\title{
Robotic urology in the United Kingdom: experience and overview of robotic-assisted cystectomy
}

\author{
Oussama Elhage $\cdot$ Declan Murphy $\cdot$ Ben Challacombe $\cdot$ \\ Peter Rimington • Mohammad S. Khan • \\ Prokar Dasgupta
}

Received: 12 April 2007 / Accepted: 5 December 2007 / Published online: 8 January 2008

(C) Springer London 2008

\begin{abstract}
In this article we look at the evolution of robotic technology in operative urology and the significant early contribution of Mr John Wickham. We explore the ergonomics of robotic technology and discuss financial issues from a British perspective. We share our clinical experience, describe the authors' robotic-assisted cystectomy technique, and conclude by exploring the patients' perception of this new treatment modality.
\end{abstract}

Keywords Robotic $\cdot$ da Vinci $\cdot$ Cystectomy

\section{Introduction}

The use of robotic-assisted laparoscopic surgery throughout the world has increased significantly in recent years and is now standard practice in many large centres. British urologists were among the first to contribute to the development of surgical robotics and embrace this new technology. Currently, robotic-assisted radical prostatectomy is the most commonly performed robotic procedure worldwide [1], and it is now gaining popularity in the United Kingdom.

O. Elhage $\cdot$ D. Murphy $\cdot$ B. Challacombe $\cdot$ P. Rimington .

M. S. Khan · P. Dasgupta $(\square)$

Department of Urology,

Guy's Hospital and King's College London School of Medicine,

1st Floor Thomas Guy House, London SE1 9RT, UK

e-mail: prokarurol@gmail.com

P. Rimington

Eastbourne General Hospital, Eastbourne, UK

\section{History}

Man's fascination with machines that are able to perform automated work is hundreds of years old, with the evidence for this scattered in the literature of several different cultures. Leonardo da Vinci probably designed the first robot, an automated knight capable of performing basic movements to entertain the guests of Leonardo's patron [2]. It was not until the last three decades of the twentieth century that robotic technology was developed for medical applications. The earliest machines were supportive robots used for transporting pharmaceuticals and medical equipment, and rehabilitation robots which provided physiotherapy to joints and assisted stroke patients with their daily tasks. In the 1980s researchers explored the potential of robotics in operative surgery. Several projects in the United States and in Europe started, some independently and some as collaborative efforts.

One of the first pioneers was John Wickham, a urologist from Guy's Hospital. He developed the first clinically useful robot in urology, the PROBOT in 1989 [3]. In the late 1980s Wickham worked on a TURP robotic frame in a joint project between the Mechanical Engineering Department at Imperial College, Guy's Hospital and the Institute of Urology in central London. The device attempted to perform an automated robotic transurethral resection of the prostate (TURP). The team felt that as the prostate is a relatively fixed organ and the procedure requires repeated similar movements, TURP was ideal for robotic control. The frame was constructed to support a six-axis Unimate Puma robot combined with a Wickham Endoscope liquidizer and aspirator. The liquidizer blade rotated at $40,000 \mathrm{rpm}$ and the system was used in initial clinical trials following successful tests on prostateshaped potatoes. These showed the PROBOT-assisted 
TURP to be not only safe, feasible and rapid, but it also provided good haemostasis when used clinically. One important concept in the design was that the tool could cut or vaporize only within a physically restricted volume, making the device intrinsically safe. Although never mass-produced, this was the first truly automated robotic device in clinical use, as opposed to the subsequent master-slave devices which technically perform roboticassisted surgery.

The master-slave systems (initially the telepresence system) were developed as a collaborative effort between the National Aeronautics and Space Administration (NASA) which had expertise in virtual reality and The Stanford Research Institute (SRI) headed by Philip Green in the 1980s [4]. Several years passed before the next generation of robotic devices became available. Computer Motion (Berkeley, CA, USA) introduced the Automated Endoscopic System for Optimal Positioning (AESOP) in the mid-1990s. This system uses voice (or pedal) control to direct the movements of a robotic arm, which usually holds a laparoscopic camera. Another robotic manipulator is the EndoAssist (Armstrong Healthcare, High Wycombe, UK), a free-standing laparoscopic camera manipulator controlled by infrared signals from a headset worn by the surgeon. It was also introduced in the 1990s and although it is considerably less expensive than the AESOP it takes up more space within the operating room.

Computer Motion introduced its ZEUS Robotic Surgical System within a year of the introduction of da Vinci by Intuitive Surgical (Sunnyvale, CA, USA). Both were the first commercially available master-slave robotic surgical systems and both had a remote station that could control laparoscopic instruments at the patient side. The ZEUS system had AESOP incorporated in its design to control the camera. Originally it only had 2-D imaging, but it was later updated to 3-D vision using polarizing glasses. Its robotic arms were attached independently to the operating table. Tremor elimination and motion scaling were possible. The combination of ZEUS with a telecommunication system allowed Prof Marescaux in New York to perform a laparoscopic cholecystectomy on a patient in Strasbourg, France. This was the first operation to be performed on a human over a long distance and was called the Lindbergh procedure [5]. In a comparative study on animals, Sung and Gill noted that ZEUS provided five degrees of freedom (DOF) at the operative site, and the learning curve and operations were longer than those for the da Vinci system; however, this study was done before the introduction of Microwrist to ZEUS systems [6]. After years of legal disputes Intuitive Surgical acquired Computer Motion in 2003 and subsequently the ZEUS system was phased out.

\section{Basic science}

The technology

The da Vinci is the most advanced master-slave system in current clinical use with the introduction of the updated da Vinci $S$ in 2006. The system has been described in detail in a previous publication of this journal and elsewhere [7, 8]. Its 3-D vision, enhanced magnification, motion scaling and more importantly the EndoWrist technology make the daVinci an easy-to-use, surgeon-friendly tool. Another potential direction for the master-slave system is true telerobotic surgery, which has many applications in military environments, space exploration and surgery in remote areas where the skill of the surgeon can be utilised without him or her being physically present at the patient side. The first telerobotic procedure was a prostate biopsy performed by an Italian group in 1995 [9]. The Lindbergh operation has already been mentioned above. Recently, Challacombe et al. [10] found that robotic kidney needle access is slower and more accurate compared to human in a randomised controlled trial of telerobotic surgery between London and Baltimore. Another application is robotic telementoring [11], which enables an experienced surgeon to direct and mentor another surgeon remotely.

\section{Ergonomics}

Laparoscopy remains at the forefront of urological minimally-invasive surgery; however, many procedures are technically demanding, with a considerable learning curve. Manipulation of long laparoscopic instruments causes a number of ergonomic problems. There is a fulcrum effect at the point of trocar insertion through the abdominal wall, where a hand movement to the right produces a counterintuitive movement to the left at the tip of the instrument within the operative field. Instruments are long and move in a cone-shaped way with the tip of the cone at the trocar insertion point on the abdominal wall. Arc-like movements of the upper extremity are necessary to produce small movements of the end effector, leading to reports of arm and neck pain among laparoscopic surgeons [12]. The pistol-type handle forces the hand into extreme positions of flexion and ulnar deviation at the wrist, and this requires more muscle contractions to perform a task compared to inline handle or open techniques [13]. During laparoscopic surgery the majority of the surgeon's movements are at the level of the hands, wrists and, to a lesser degree, the shoulders. The rest of the body is in an upright position, which may be responsible for the neck and back discomfort associated with laparoscopy [14]. The current laparoscopic instrumentation allows only four DOF. In contrast the EndoWrist in the da Vinci system has seven DOF at the tip 
of the instrument. The hand of the Robonaut developed by NASA has a total of $14 \mathrm{DOF}$ [15], and the human hand is of course the most dexterous, with 36 DOF [16]. This limitation of laparoscopy results in a restriction of the manoeuvrability of the surgeon and contributes to the ergonomic awkwardness associated with complex laparoscopic procedures, especially those involving suturing. Normal 3-D vision is lost due to the monoscopic camera system, reducing the depth of field and causing eyestrain for the surgeon [17]. The cumulative effect of these problems is to increase overall fatigue and stress and restrict the number of minimally invasive surgical procedures that can be performed by one surgeon in a given operative session [18]. The master-slave robotic systems may help resolve some of the ergonomic obstacles described above. With the da Vinci ${ }^{\mathrm{TM}}$ system, the surgeon is seated at a console remote from the patient, providing a much more ergonomic posture than that of the traditional patient-side surgeon. The fingertip controls allow "intuitive" rather than "fulcrum"-type control over the laparoscopic instruments, which may help to reduce fatigue in the upper extremity and neck. The restoration of increased DOF compared to the four DOF of conventional laparoscopy may offer benefits for complex laparoscopic tasks [19]. This may also decrease the excessive wrist strain during laparoscopy. 3-D stereoscopic vision can also provide advantages over the 2-D monoscopic vision of conventional laparoscopic systems [20]. We are currently comparing the impact of the physical activities of both techniques on surgeons. Standard tasks are performed using open, laparoscopic and robotically assisted techniques. Electromyographic (EMG) sensors record muscular activity; motion capture cameras capture postural variation. An analysis of the data obtained will allow objective comparison of these techniques and will help us to understand their impact on surgeons [21].

\section{Financial hurdles}

There are currently 400 da Vinci systems the United States compared to seven in the UK; this corresponds to one system per 750,000 of the US population compared to one in 15 million in the UK. Differences in the structures of the health systems in both countries partially account for this discrepancy. In the UK at present only large training centres are able to purchase da Vinci systems, often with the support of large charitable organisations [22]; however, there is increasing interest from the private sector. The current cost of the da Vinci system is $£ 700,000$ (\$1.2 million). This is not the only cost; annual maintenance is $£ 70,000$ $(\$ 138,000)$. The instrument cost of a typical roboticassisted prostatectomy case at Guy's Hospital, London is around $£ 1,000(\$ 1,700)$, with an additional $£ 650(\$ 1,100)$ for consumables, compared to $£ 950(\$ 1,600)$ for laparo- scopic radical prostatectomy and $£ 670$ for open radical prostatectomy [23]. Payment by results is a new system implemented recently by the British government, and it is changing the landscape in health care. This makes length of stay (LOS) a key productivity and efficiency variable for National Health Service (NHS) hospitals. A one-day stay in hospital in the UK costs $£ 214$ (\$360)—£225 (\$380) for general wards-and ranges between $£ 1,300(\$ 2,200)$ and $£ 1,700(\$ 2,900)$ for intensive care beds [24]. This may prove vital for robotic-assisted operations, as early discharge from hospital is a possible advantage.

In their study, Link et al. [25] suggest that depreciation and maintenance costs can be minimised if the number of robotic cases is increased. In another study, Scales et al. [26] indicated that while prolonged operative time increases the cost exponentially, increasing the number of robotic-assisted prostatectomies to ten per week can be cost-equivalent to open prostatectomy.

\section{Training of a team}

Another essential task to overcome in addition to financial problems is to train a robotic-assisted surgical team. In our unit, the members of our robotic group (including surgeons and nurses) were initially trained in a da Vinci dry lab setting and subsequently in a cadaveric lab in Paris. The team travelled to the Vatikutti Institute in Detroit to observe live cases. Later an experienced urologist from the Vatikutti Institute locally mentored our team in our initial cases in the UK [22]. Other teams had similar training [27].

\section{The clinical experience}

Several centres in the UK started robotic surgery programmes shortly after master-slave systems became available commercially. This trend first started with cardiac surgery. Deeba and Darzi [28] reported 102 cases of robotic-assisted cardiac surgery. Undre and Darzi from the same institution reported robotic-assisted Heller cardiomyotomy $(n=5)$ [29] and robotic-assisted adrenalectomy $(n=2)$ [30]. Subsequently the technology was adopted mainly by urologists. To date there are seven centres in the UK that have started robotic-assisted surgery programmes. The trend in the UK is the same as in the rest of the world, robotic-assisted prostatectomy (RAP) is the most commonly performed procedure. In their first 50 RAP cases, Mayer et al. [31] reported an operative time of nearly 370 min, a transfusion rate of $12 \%$ with median blood loss of $700 \mathrm{ml}$, a $36 \%$ complication rate including two rectal injuries, a hospital stay of four days, and $22 \%$ positive margins. Results are mostly comparable to published early 
experiences [32]. Two other series of the first 100 cases showed decreased operative time, blood loss, and positive margins [33, 34]. Many other urological procedures have been performed. Murphy et al. [35] reported their experiences in robotic renal surgery using da Vinci systems. They have performed robotic-assisted pyeloplasty $(n=15)$, simple nephrectomy $(n=2)$, radical nephrectomy $(n=1)$, nephrouretrectomy $(n=2)$ and live donor nephrectomy $(n=4)$ [35]. The mean operative time was $215 \mathrm{~min}$, blood loss $75 \mathrm{ml}$, complication rate $2.7 \%$ and hospital stay 2.9 days. Three of the nephrectomies were performed retroperitoneally, and four using hand-assisted approach. Complications included laparotomy for liver injury. Dasgupta and Khan [22] from the same group reported roboticassisted colposuspension. Table 1 summarises the series of robotic cases in the UK.

\section{Robotic-assisted cystectomy}

Cystectomy is the most effective treatment for invasive bladder cancer, uncontrollable superficial cancer and refractory carcinoma in situ; however, it can be a morbid operation with high risk of complications and slow recovery [36]. A minimally invasive approach was shown to speed recovery [37], decrease blood loss and postoperative pain [38] and has acceptable functional and oncological outcome [39]. However laparoscopic cystectomy (LC) is still a lengthy procedure and requires extensive skills which limit this technique to a few centres [40]. Robotic-assisted surgery has the potential to overcome some of the laparoscopic difficulties. Robotic-assisted cystectomy (RAC) was first described by Menon et al. [41] in 2003 using the da Vinci system, the Vattikuti technique. Access is through six ports utilizing a transperitoneal approach. An extracorporeal ileal conduit or orthotopic bladder is fashioned, with the robot redocked for anastomosis in the case of neobladder. A team from Sweden perform all of the procedure

Table 1 Robotic-assisted cases in the UK

\begin{tabular}{lcll}
\hline Authors & Year & Robotic-assisted cases & $N$ \\
\hline Undre et al. [29] & 2004 & Heller cardiomyotomy & 5 \\
Undre et al. [30] & 2004 & Adrenalectomy & 2 \\
Rose et al. [46] & 2006 & Retroperitoneal & 2 \\
& & nephrouretrectomy & \\
Deeba et al. [47] & 2006 & Cardiac surgery & 102 \\
Mayer et al. [48] & 2006 & Prostatectomy & 50 \\
Mruphy et al. [35] & 2007 & Pyeloplasty & 15 \\
& & Nephrectomy & 2 \\
& & Radical nephrectomy & 1 \\
& & Live donor nephrectomy & 4 \\
Dasgupta et al. [49] & 2007 & Cystectomy & 19 \\
\hline
\end{tabular}

intracorporeally [42]. Rhee et al. [43] dissect lymph nodes through an open incision. RAC is still only performed by a limited number of centres worldwide; however, it is gaining wider acceptance. Early series results reflect the advantages of laparoscopic surgery: less blood loss, early recovery and adequate cancer control [43-45]. However larger series and controlled trials are needed. There is still a lack of long-term oncological follow-up. Recent series are summarized in Table 5. Here the authors report their series-the first in the UK and one of the first in Europe. Our team consists of two surgeons experienced in open cystectomy and one experienced in robotic-assisted surgery. Our technique is a modification of the Vattikuti technique.

\section{The Guy's operative technique}

Patients have bowel preparation and overnight intravenous fluids. Patients above 60 are digitalised as recommended by urologists experienced in open cystectomy, to prevent atrial fibrillation. They are placed in the extended lithotomy position with a $45^{\circ}$ Trendelenburg tilt. A disposable sigmoidoscope is introduced per rectum in male patients and a methylene-blue-soaked swab per vaginum in female patients. After catheterisation, a six-port transperitoneal approach is used. Each procedure involves three surgeons-one at the console and one on each side of the patient.

\section{Posterior dissection}

The distal ends of ureters are sent for frozen section analysis. An inverted U-shaped incision is made in the peritoneum of the Pouch of Douglas. The posterior layer of Denonvillier's fascia is then incised in the midline and the plane between the rectum and the prostate developed. In females the ovarian vessels are controlled and divided. The plane between the rectum and uterus is developed and the uterine arteries controlled.

\section{Lateral dissection}

Dissection is continued medial to the external iliac veins to carefully preserve the obturator nerves and expose the lateral pelvic wall. This delineates the lateral pedicles to the bladder (and uterus in females), which are controlled with the ACE Harmonic ${ }^{\mathrm{TM}}$ scalpel (Ethicon Endosurgery, Livingston, UK).

\section{Anterior dissection}

The bladder is filled with $200 \mathrm{ml}$ of formal saline to aid identification and dropped by an inverted $U$ incision to include the urachus. The endopelvic fascia is opened and 
the dorsal vein controlled by a stitch. Nerve sparing is performed for potent male patients. The distal urethral margin is sent for frozen section. In females the urethra is dissected fully to the external meatus and the posterior vaginal fornix opened. The previously placed methylene blue swab becomes visible, indicating that the correct plane had been entered. The lateral vaginal walls are transected. The cystectomy specimens are secured in a bag for later retrieval. Leakage of carbon dioxide from the vagina is reduced by a waterproof dressing applied externally. The vagina is then closed longitudinally by continuous intracorporeal suturing with 2-0 vicryl.

\section{Lymphadenectomy and transposition of left ureter}

Using robotic bipolar forceps and scissors, careful bilateral lymphadenectomy is performed. The limits of the dissection are the genitofemoral nerve laterally, the bifurcation of the common iliac artery proximally and the node of Cloquet distally. Care is taken to preserve the obturator nerve. The lymph nodal packs are placed in separately marked laparoscopic sacks. An Endoloop ${ }^{\mathrm{TM}}$ (Ethicon Endosurgery, Livingston, UK) is applied on the distal end of the left ureter which is then transposed under the sigmoid mesocolon to the left by pulling the Endoloop ${ }^{\mathrm{TM}}$ through. The distal ends of the ureters are held together with a laparoscopic grasper introduced through the left-sided 5-mm assistant port.

\section{Urinary diversion}

All diversions are performed extracorporeally. For ileal conduits, a $15-\mathrm{cm}$ segment of ileum about $15 \mathrm{~cm}$ proximal to the ileocaecal junction is held in laparoscopic graspers introduced through the most lateral right-sided $10-\mathrm{mm}$ port. The robot is undocked. The previously bagged bladder and lymph nodal specimens are extracted through a 5-7 cm incision. In thin patients this should be an appendix musclesplitting incision made by extending a lateral port, while in overweight patients (BMI $>30 \mathrm{Kg} / \mathrm{m}^{2}$ ) a subumbilical midline incision is preferred for easier left ureteric access. The graspers holding the ureters and ileal segment are brought to the surface through this incision. The ileal loop is isolated on its mesentery, bowel continuity restored with staplers and the mesenteric window closed. Ureteroileal anastomosis is performed over $8 \mathrm{~F}$ feeding tubes using a Wallace I technique. The distal end of the conduit is fashioned as a stoma at a previously marked site on the abdominal wall. A sump drain is introduced into the conduit to prevent any anastomotic pressure and leak from subsequent stomal oedema. Studer pouches are created through lower midline incisions and anastomosed to the urethral stump by six robotically placed 3-0 monocryl sutures. A $20 \mathrm{~F}$ drain is placed in the pelvis. The port sites and wounds are closed with absorbable sutures. A litre of icodextrin (Adept, ML Pharmaceuticals, Warrington, UK) is instilled into the abdomen and drained after an hour to reduce the risk of bowel adhesions.

\section{Post-operative care}

Initially all of our patients were electively managed in a high-dependency unit, as this was a new procedure in our centre. The nasogastric tube is removed next morning and oral liquids started as tolerated. Early mobilisation and chest physiotherapy are encouraged. Most patients are discharged with their pelvic drains and ureteric catheters in situ, which are removed at 2-3 weeks. Patients are seen again at six weeks and have an abdominal ultrasound at three months, CT scans at six months and then at sixmonthly intervals. At these visits they have clinical examination and assessment of serum haemoglobin, electrolytes, creatinine, chloride and bicarbonate.

\section{Cases and outcome}

We have performed 19 cases. Patients diagnosed with T4 bladder cancer and those having previous pelvic radiotherapy or abdominal surgery were excluded. All procedures were completed without conversion; two patients had Studer pouches fashioned. Bowel function returned on day 1 except in the pouches. Patient satisfaction was high: 30 (29-31) out of 32 on a validated patient satisfaction questionnaire (CSQ-8). Four patients had complications. One patient had rectal injury in a T3 adenocarcinoma such that the urethra needed colostomy; one of the Studer pouch patients developed urethrovesical stricture and was treated successfully with endoscopic dilatation. A patient had port site bleeding which needed blood transfusion, and one patient developed incisional hernia and was surgically

Table 2 Patient demographics of robotic-assisted radical cystectomy at Guy's Hospital

\begin{tabular}{ll}
\hline Patients & \\
\hline$N$ & 19 \\
Male & 13 \\
Female & 6 \\
Age & $62(57-77)^{\mathrm{a}}$ \\
BMI & $>30$ in ten patients
\end{tabular}

\section{Indications}

Muscle invasive TCC $\quad 10$

Refractory CIS 4

G3T1 TCC 4

Urethral adenocarcinoma $\quad 1$

$\overline{B M I \text { body mass index, } T C C \text { transitional cell carcinoma, } C I S \text { carcinoma }}$ in situ

a Median value 
Table 3 Operative details of robotic-assisted radical cystectomy at Guy's Hospital

\begin{tabular}{ll}
\hline & Median values \\
\hline Operative time (min) & $320(295-510)$ \\
Docking time (min) & $6(2-9)$ \\
Blood loss (ml) & $150(100-1,150)$ \\
Clear surgical margins & $100 \%$ \\
Hospital stay (d) & $10(6-22)$ \\
Full recovery (w) & $5(4-12)$ \\
Lymph node dissection & $16(6-25)$ \\
Conversion & None \\
\hline
\end{tabular}

Min minutes, $m l$ millilitre, $d$ days, $w$ weeks

Table 4 Functional and oncological outcome in robotic-assisted radical cystectomy at Guy's Hospital

\begin{tabular}{ll}
\hline Complication (21\%) & $\begin{array}{c}\text { One port site bleeding needing blood } \\
\text { transfusion } \\
\text { One rectal injury in a case with urethral } \\
\text { adenocarcinoma, colostomy } \\
\text { One incisional hernia, repaired } \\
\text { One urethrovesical stricture } \\
\text { (Studer pouch), dilatation }\end{array}$ \\
& \\
Follow-up (30 months) & $94 \%(18 / 19)$ \\
Overall survival & $89 \%(17 / 19)$ \\
Disease-free survival &
\end{tabular}

repaired. There were no perioperative deaths. On followup, two patients developed metastasis, one of which has died. Patient demographics, operative details, and outcome are all summarized in Tables 2 and 3, 4 and 5.

\section{Patient experience}

Little is known about the public perception of robotic surgery in the United Kingdom. In the first year of setting up our robotic programme, we had eight patient enquiries from across the UK. The referrals largely came from colleagues in other centres. This was surprising, and reflected some early reservations amongst patients. However, subsequent widespread media coverage has increased patient awareness of the possibilities offered by robotic surgery, and has led to a market-driven demand for robotic surgery for some procedures. At Guys we receive public exposure via a daytime hospital programme which is broadcast by the BBC. "City Hospital" shows real-life medical procedures, some broadcast live. As a result we often get queries about our techniques and the availability of our services. In addition, we have held a robotics "patient day". On this occasion patients and their partners were invited to attend presentations and discussions on the robotic programme and give

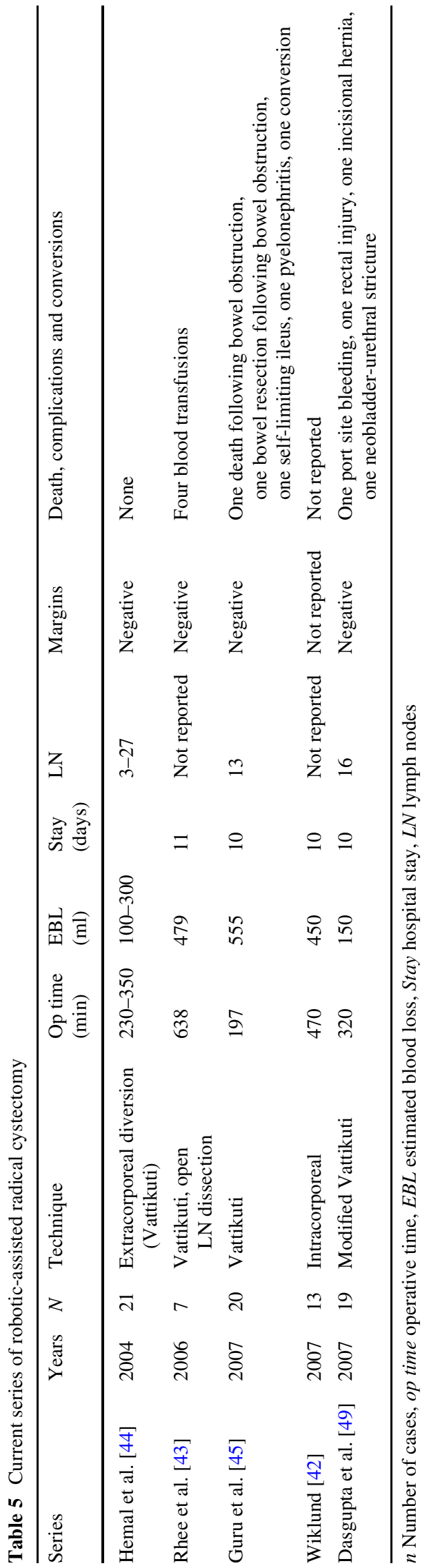


feedback on their experiences. We addressed two main areas - their expectations of the service, and what level of information should be available to patients undergoing robotic surgery. Many important topics were discussed, some regarding the good experiences patients had regarding the video availability of the procedures, leaflets and postoperative pain control. Another issue was the difficulty involved in patients obtaining access to robotic surgery due to the paucity of machines in the UK. Most patients preferred to receive counselling about the robot from a specialist nurse with a fixed contact telephone number in case of any difficulty.

\section{Discussion}

Robotic surgery presents many appealing advantages to both surgeons and patients. It offers all of the benefits of laparoscopic surgery; in addition it provides a steady tremor-free scaled motion. The intuitive movements and the enhanced DOF with the 3-D stereoscopic vision suggest an advantage over laparoscopic surgery. The early functional outcomes of robotic radical prostatectomy are promising; however, most of the comparative studies are from single institutions, and lack a high level of evidence [32].

The high cost of purchasing and maintaining the instruments of the robotic system is one of its many disadvantages. The absence of haptic feedback remains an important issue, but this technology may well be introduced in the near future. The current da Vinci system is still sizeable and requires a team of trained staff to set it up over a lengthy time period. The availability of the robotic systems to only a limited number of centres reduces surgical training opportunities, and this is particularly obvious in Britain. However, overall, robotic surgery in the UK is evolving, with public acceptance and awareness increasing steadily.

\section{References}

1. Peplinski R (2006) Past, present and future of the da Vinci robot. 2nd UK Robotic Urology Course. Guy's Hospital, London

2. Rosheim ME (2006) Leonardo's lost robots. Springer, Berlin

3. Challacombe BJ, Khan MS, Murphy D, Dasgupta P (2006) The history of robotics in urology. World J Urol 24(2):120-127

4. Satava RM (2002) Surgical robotics: the early chronicles: a personal historical perspective. Surg Laparosc Endosc Percutan Tech 12(1):6-16

5. Marescaux J, Leroy J, Gagner M, Rubino F, Mutter D, Vix M et al. (2001) Transatlantic robot-assisted telesurgery. Nature 413:379-380

6. Sung GT, Gill IS (2001) Robotic laparoscopic surgery: a comparison of the DA Vinci and Zeus systems. Urology 58(6):893-898

7. Thaly R, Shah K, Patel VR (2007) Applications of robots in urology. J Robot Surg 1(1):3-17

8. Murphy D, Challacombe B, Khan MS, Dasgupta P (2006) Robotic technology in urology. Postgrad Med J 82(973):743-747
9. Rovetta A, Sala R (1995) Robotics and telerobotics applied to a prostate biopsy on a human patient. Proceedings of the Second Symposium on Medical Robotics and Computer Assisted Surgery (MRCAS '95). Wiley, New York, p 104

10. Challacombe B, Patriciu A, Glass J, Aron M, Jarrett T, Kim F et al. (2005) A randomized controlled trial of human versus robotic and telerobotic access to the kidney as the first step in percutaneous nephrolithotomy. Comput Aided Surg 10(3):165-171

11. Challacombe B, Kavoussi L, Patriciu A, Stoianovici D, Dasgupta $P$ (2006) Technology insight: telementoring and telesurgery in urology. Nat Clin Pract Urol 3(11):611-617

12. Nguyen NT, Ho HS, Smith WD, Philipps C, Lewis C, De Vera RM et al. (2001) An ergonomic evaluation of surgeons' axial skeletal and upper extremity movements during laparoscopic and open surgery. Am J Surg 182(6):720-724

13. Berguer R, Gerber S, Kilpatrick G, Beckley D (1998) An ergonomic comparison of in-line vs pistol-grip handle configuration in a laparoscopic grasper. Surg Endosc 12(6):805-808

14. Berguer R, Rab GT, bu-Ghaida H, Alarcon A, Chung J (1997) A comparison of surgeons' posture during laparoscopic and open surgical procedures. Surg Endosc 11(2):139-142

15. NASA (2007) Robonaut hands. National Aeronautics and Space Administration (NASA), Washington, DC

16. Patkin M, Isabel L (1995) Ergonomics, engineering and surgery of endosurgical dissection. J R Coll Surg Edinb 40(2):120-132

17. Hemal AK, Srinivas M, Charles AR (2001) Ergonomic problems associated with laparoscopy. J Endourology 15(5):499-503

18. Berguer R, Smith WD, Chung YH (2001) Performing laparoscopic surgery is significantly more stressful for the surgeon than open surgery. Surg Endosc 15(10):1204-1207

19. Talamini M, Campbell K, Stanfield C (2002) Robotic gastrointestinal surgery: early experience and system description. J Laparoendosc Adv Surg Tech A 12(4):225-232

20. Jourdan IC, Dutson E, Garcia A, Vleugels T, Leroy J, Mutter D et al. (2004) Stereoscopic vision provides a significant advantage for precision robotic laparoscopy. Br J Surg 91(7):879-885

21. Elhage O, Murphy D, Challacombe B, Shortland A, Dasgupta P (2007) Ergonomics in minimally invasive surgery. Int J Clin Prac 61(2):186-188

22. Dasgupta P, Hemal A, Rose K 2005) Robotic urology in the UK: establishing a programme and emerging role. BJU Int 95(6):723724

23. Urology Department (2007) Guy'sHospital, London, UK

24. DoH (2006) NHS reference costs 2005-2006. Department of Health, London

25. Link RE, Bhayani SB, Kavoussi LR (2006) A prospective comparison of robotic and laparoscopic pyeloplasty. Ann Surg 243(4):486-491

26. Scales CD Jr, Jones PJ, Eisenstein EL, Preminger GM, Albala DM (2005) Local cost structures and the economics of robot assisted radical prostatectomy. J Urol 174(6):2323-2329

27. Kaul SA, Peabody JO, Shah N, Neal D, Menon M (2006) Establishing a robotic prostatectomy programme: the impact of mentoring using a structured approach. BJU Int 97(6):1143-1144

28. Deeba S, Aggarwal R, Sains P, Martin S, Athanasiou T, Casula R et al. (2006) Cardiac robotics: a review and St Mary's experience. Int J Med Robot 2(1):16-20

29. Undre S, Moorthy K, Munz Y, Aggarwal R, Hance J, Rockall T et al. (2004) Robot-assisted laparoscopic Heller cardiomyotomy: preliminary UK results. Dig Surg 21(5-6):396-400

30. Undre S, Munz Y, Moorthy K, Martin S, Rockall T, Vale J et al. (2004) Robot-assisted laparoscopic adrenalectomy: preliminary UK results. BJU Int 93(3):357-359

31. Mayer EK, Winkler MH, Aggarwal R, Karim O, Ogden C, Hrouda $D$ et al (2006) Robotic prostatectomy: the first UK experience. Int J Med Robot 2(4):321-328 
32. Ficarra V, Cavalleri S, Novara G, Aragona M, Artibani W (2007) Evidence from robot-assisted laparoscopic radical prostatectomy: a systematic review. Eur Urol 51(1):45-56

33. Goldstraw MA, Corbishley C, Besarani D, Anderson CJ, Dasgupta P, Kirby RS (2007) A comparative study of 100 robotic assisted laparoscopic prostatectomy (RALP) versus 100 open retropubic prostatectomy. BJUI 99(s4):35

34. Tyreman N, Shah N, Basnett G, Neal DE (2007) Learning curve during robotic-assisted radical prostatectomy. BJU Int 99(s4):36

35. Murphy D, Challacombe B, Olsburgh J, Calder F, Mamode N, Khan MS et al. (2007) Ablative and reconstructive robotic-assisted laparoscopic renal surgery. IJCP (in press)

36. Stein JP, Lieskovsky G, Cote R, Groshen S, Feng AC, Boyd S et al. (2001) Radical cystectomy in the treatment of invasive bladder cancer: long-term results in 1,054 patients. J Clin Oncol 19(3):666-675

37. Basillote JB, Abdelshehid C, Ahlering TE, Shanberg AM (2004) Laparoscopic assisted radical cystectomy with ileal neobladder: a comparison with the open approach. J Urol 172(2):489-493

38. Cathelineau X, Arroyo C, Rozet F, Barret E, Vallancien G (2005) Laparoscopic assisted radical cystectomy: the montsouris experience after 84 cases. Eur Urol 47(6):780-784

39. Haber GP, Gill IS (2007) Laparoscopic radical cystectomy for cancer: oncological outcomes at up to 5 years. BJU Int 100(1):137-142

40. Hrouda D, Adeyoju AA, Gill IS (2004) Laparoscopic radical cystectomy and urinary diversion: fad or future? BJU Int 94(4):501-505

41. Menon M, Hemal AK, Tewari A, Shrivastava A, Shoma AM, El-Tabey NA et al. (2003) Nerve-sparing robot-assisted radical cystoprostatectomy and urinary diversion. BJU Int 92(3):232-236
42. Wiklund PN (2007) Robotic assisted radical cystectomy: technical aspects. In: 2007 World Robotic Urology Symposium, Columbus, OH, 26-27 April 2007

43. Rhee JJ, Lebeau S, Smolkin M, Theodorescu D (2006) Radical cystectomy with ileal conduit diversion: early prospective evaluation of the impact of robotic assistance. BJU Int 98(5):1059-1063

44. Hemal AK, bol-Enein H, Tewari A, Shrivastava A, Shoma AM, Ghoneim MA et al (2004) Robotic radical cystectomy and urinary diversion in the management of bladder cancer. Urol Clin North Am 31(4):719-729

45. Guru KA, Kim HL, Piacente PM, Mohler JL (2007) Robot-assisted radical cystectomy and pelvic lymph node dissection: initial experience at Roswell Park Cancer Institute. Urology 69(3):469_ 474

46. Rose K, Khan S, Godbole H, Olsburgh J, Dasgupta P (2006) Robotic assisted retroperitoneoscopic nephroureterectomy-first experience and the hybrid port technique. Int $\mathrm{J}$ Clin Pract 60(1):12-14

47. Deeba S, Aggarwal R, Sains P, Martin S, Athanasiou T, Casula R et al. (2006) Cardiac robotics: a review and St Mary's experience. Int J Med Robot 2(1):16-20

48. Mayer EK, Winkler MH, Aggarwal R, Karim O, Ogden C, Hrouda D et al. (2006) Robotic prostatectomy: the first UK experience. Int J Med Robot 2(4):321-328

49. Dasgupta P, Rimington P, Murphy D, Challacombe BJ, O'Brien TS, Khan MS (2007) Robot-assisted radical cystectomy for bladder cancer and 2 year follow-up. In: Laparoscopic/Robotics Session, British Association of Urological Surgeons Annual Meeting, Glasgow, UK, 18-22 June 2007 